\title{
PRELIMINARY ELEMENTS OF MINOR PLANET 1922 MZ (Y. O. 5)
}

Minor Planet $1922 \mathrm{MZ}$ was discovered by Van Biesbroeck on November 23, 1922. Five observations were secured in November and December, but it could not be found in January. An orbit was computed from the observations of November 24, December 15, and December 22 and an ephemeris was forwarded to Van Biesbroeck. Owing to the proximity of the Moon, and to cloudy weather he was unable to observe the asteroid during February, but on March 10 it was rediscovered about 20 minutes of arc from the predicted position.

The following observations have been furnished by Van Biesbroeck:

$\begin{array}{rlrrrrr} & & a(1925.0) & & \delta(1925.0) \\ 1922 & \text { Nov. } 23.836 & 4^{\mathrm{h}} & 37^{\mathrm{m}} & 18^{\mathrm{s}} & +36^{\circ} & 38{ }^{\prime} 1 \\ & \text { Nov. } 24.769 & & 36 & 12 & 36 & 38.1 \\ \text { Dec. } 15.840 & & 12 & 22 & 35 & 48.8 \\ & \text { Dec. } 22.707 & & 06 & 27 & 35 & 17.8 \\ 1923 & \text { Mar. } 10.595 & 4 & 42 & 26 & +31 & 16.7\end{array}$

The preliminary elements and constants for the equator 1922.0 are as follows:

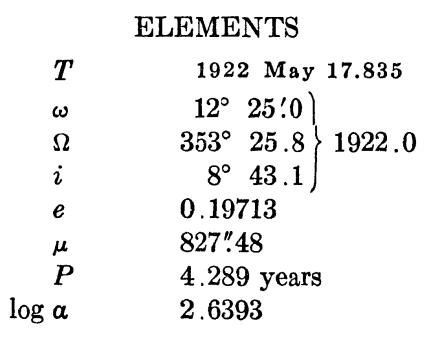

CONSTANTS FOR THE EQUATOR 1922.0

$x=r(9.99993) \sin \left(95^{\circ} 55.4+v\right)$

$y=r(9.92791) \sin \left(5^{\circ} 18.0+v\right)$

$z=r(9.72575) \sin \left(7^{\circ} 30.4+v\right)$

\section{RESIDUALS}

$\begin{array}{llrr} & & \Delta a \cos \delta & \Delta \delta \\ 1922 & \text { Nov. } 23.836 & +0 ! 1 & -0 ! 2 \\ & \text { Nov. } 24.769 & +0.2 & -0.2 \\ & \text { Dec. } 15.840 & 0.0 & 0.0 \\ & \text { Dec. } 22.707 & 0.0 & 0.0 \\ 1923 & \text { Mar. } 10.595 & -21.0 & +4.4\end{array}$

C. D. Shane.

J. A. Pearce.

Berkeley Astronomical Department, 1923 March 16

\section{OBSERVATIONS OF COMET $1922 c$ (BAADE)}

\begin{tabular}{|c|c|c|c|c|c|c|c|c|c|c|c|c|c|c|c|c|c|c|}
\hline \multirow{2}{*}{$\begin{array}{l}\text { Date } \\
1922\end{array}$} & \multirow{3}{*}{$\begin{array}{l}\text { Gr. } \\
16^{\mathrm{h}}\end{array}$} & \multirow{3}{*}{$\begin{array}{l}\mathrm{M} . \\
27^{\mathrm{m}}\end{array}$} & \multirow{3}{*}{$\begin{array}{l}\mathrm{T} . \\
48^{s}\end{array}$} & \multirow{3}{*}{$\begin{array}{c}\text { Star } \\
1\end{array}$} & \multirow{2}{*}{\multicolumn{2}{|c|}{$\begin{array}{l}\text { No. of } \\
\text { Comp. }\end{array}$}} & \multicolumn{3}{|c|}{ Comet-Star } & \multicolumn{6}{|c|}{ Comet's Apparent } & \multicolumn{2}{|c|}{$\log \rho \Delta$} & \multirow{3}{*}{$\begin{array}{l}\text { Observer } \\
\text { Aitken }\end{array}$} \\
\hline & & & & & & & $\Delta a$ & & $\Delta \delta$ & & a & & & $\delta$ & & $a$ & $\delta$ & \\
\hline Oct. 23 & & & & & 10 & 10 & $+10: 39$ & $-3^{\prime}$ & $17^{\prime \prime .5}$ & $19^{\mathrm{h}}$ & $55^{\mathrm{m}}$ & $56: 73$ & $+36^{\circ}$ & $34^{\prime}$ & $33 " .5$ & 9.555 & 9.991 & \\
\hline Oct. 24 & 15 & 15 & 54 & 2 & 10 & 10 & +12.99 & -3 & 42.5 & 19 & 58 & 04.55 & +36 & 18 & 10.8 & 9.308 & 9.596 & Neubauer \\
\hline Oct. 28 & 17 & 37 & 58 & 3 & 10 & 10 & -7.86 & + & 08.5 & 20 & 07 & 27.11 & +35 & 07 & 09.2 & 9.684 & 0.341 & Shane \\
\hline Oct. 28 & 18 & 02 & 32 & 4 & 10 & 10 & -5.46 & - & 10.0 & 20 & 07 & 29.51 & +35 & 06 & 50.7 & 9.710 & 0.410 & Aitken \\
\hline Nov. 11 & 18 & 15 & 00 & 5 & 10 & 10 & -9.30 & -1 & 23.1 & 20 & 41 & 08.88 & +31 & 12 & 49.3 & 9.716 & 0.537 & Pearce \\
\hline Nov. 11 & 19 & 22 & 00 & 6 & 10 & 10 & -2.44 & -2 & 11.2 & 20 & 41 & 15.74 & +31 & 12 & 01.2 & 9.737 & 0.652 & Pearce \\
\hline $\begin{array}{c}\text { Nov. } 17 \\
1923\end{array}$ & 17 & 10 & 45 & 7 & 10 & 10 & +6.15 & -2 & 42.4 & 20 & 55 & 46.66 & +29 & 25 & 54.2 & 9.660 & 0.451 & Pearce \\
\hline Jan. 6 & 14 & 55 & 46 & 9 & 10 & 10 & -8.04 & -2 & 04.7 & 22 & 57 & 50.08 & +19 & 08 & 00.1 & 9.549 & 0.533 & Pearce \\
\hline Jan. & 17 & 32 & 39 & 10 & 10 & 10 & +3.03 & + & 56.3 & 22 & 58 & 05.38 & +19 & 07 & 09.6 & 9.693 & 0.684 & Pearce \\
\hline Jan. 6 & 17 & 59 & 17 & 11 & 10 & 10 & +4.74 & + & 53.1 & 22 & 58 & 07.04 & 19 & 07 & 06.1 & 9.694 & 0.698 & Jacobsen \\
\hline Jan. 13 & 17 & 30 & 15 & 12 & 10 & 10 & -10.90 & + & 41.1 & 23 & 13 & 52.89 & 18 & 17 & 40.5 & 9.690 & 0.690 & Jacobsen \\
\hline *Feb. 17 & 15 & 20 & 40 & 13 & 10 & 8 & -11.02 & -2 & 20.7 & 0 & 26 & 58.10 & 15 & 58 & 32.8 & 9.656 & 0.644 & Jacobsen \\
\hline
\end{tabular}

\section{MEAN PLACES OF COMPARISON STARS FOR 1922.0}

\begin{tabular}{|c|c|c|c|c|c|c|c|c|c|}
\hline Star & & & a & $\begin{array}{c}\text { Red. to } \\
\text { App. place }\end{array}$ & & $\delta$ & & $\begin{array}{c}\text { Red. to } \\
\text { App. place }\end{array}$ & Authority \\
\hline 1 & $19^{\mathrm{h}}$ & $55^{\mathrm{m}}$ & $44: 52$ & $+1: 82$ & $+36^{\circ}$ & $37^{\prime}$ & $18 " 4$ & $+32 " 6$ & Potsdam Photo. plate 920, Nr. 442 \\
\hline 2 & 19 & 57 & 49.74 & +1.82 & +36 & 21 & 20.7 & +32.6 & A. G. Lund 8927 \\
\hline 3 & 20 & 07 & 33.14 & +1.83 & +35 & 06 & 28.3 & +32.4 & Potsdam Photo. plate 615, Nr. 245 \\
\hline 4 & 20 & 07 & 33.14 & +1.83 & +35 & 06 & 28.3 & +32.4 & Potsdam Photo plate 615, Nr. 245 \\
\hline $\mathbf{5}$ & 20 & 41 & 16.24 & +1.94 & +31 & 13 & 40.1 & +32.3 & A. G. Leiden 8445 \\
\hline 6 & 20 & 41 & 16.24 & +1.94 & +31 & 13 & 40.1 & +32.3 & A. G. Leiden 8445 \\
\hline 7 & 20 & 55 & 38.53 & +1.98 & +29 & 28 & 04.7 & +31.9 & Micrometer Comparison with (8) \\
\hline 8 & 20 & 55 & 44.65 & +1.98 & +29 & 30 & 59.9 & +31.9 & A. G. Cambridge 11980 \\
\hline
\end{tabular}


MEAN PLACES OF COMPARISON STARS FOR 1923.0

\begin{tabular}{cccccccccc} 
Star & \multicolumn{4}{c}{$a$} & \multicolumn{3}{c}{$\begin{array}{c}\text { Red. to } \\
\text { App. place }\end{array}$} & \multicolumn{3}{c}{$\begin{array}{c}\text { Red. to } \\
\text { App. place }\end{array}$} & Authority \\
9 & $22^{\mathrm{h}}$ & $57^{\mathrm{m}}$ & 58.65 & -0.53 & $+19^{\circ}$ & $09^{\prime}$ & $58 \prime \prime 7$ & +6.1 & A. G. Berlin A 9412 \\
10 & 22 & 58 & 02.88 & -0.53 & +19 & 06 & 07.2 & +6.1 & A. G. Berlin A 9413 \\
11 & 22 & 58 & 02.88 & -0.53 & +19 & 06 & 07.2 & +6.1 & A. G. Berlin A 9413 \\
12 & 23 & 14 & 04.29 & -0.51 & +18 & 16 & 54.8 & +4.5 & A. G. Berlin A 9516 \\
13 & 0 & 27 & 09.65 & -0.52 & +16 & 00 & 55.7 & -2.2 & A. G. Berlin A 131
\end{tabular}

With the exception indicated above, all the observations were made with the 36 -inch refractor, the measures of $\Delta a$ and $\Delta \delta$ in every case being obtained directly with the micrometer. Corrections for differential refraction have been applied whenever they were appreciable. Acknowledgement is made to Mr. T. S.
Jacobsen for reducing his observations and duplicating part of the computation.

J. A. Pearce.

Lick Observatory,

May 24, 1923.

Issued August 8, 1923. 